\title{
Packet Wavelet Decomposition: An Approach For Atrial Activity Extraction
}

\author{
C Sánchez ${ }^{1}, \mathrm{~J} \mathrm{Millet}^{2}$, J J Rieta ${ }^{2}$, F. Castells ${ }^{2}, \mathrm{~J}_{\text {Ródenas }}{ }^{1}$, R. Ruiz-Granell ${ }^{3}$, V. Ruiz ${ }^{3}$ \\ ${ }^{1}$ DIEEA. E.U.P. Cuenca, Universidad de Castilla-La Mancha, Spain \\ ${ }^{2}$ BET. DIEo. Universidad Politécnica Valencia, Spain \\ ${ }^{3}$ Hospital Clínico Universitario, Valencia, Spain
}

\begin{abstract}
Detection of atrial activity (AA) is quite important in the study and monitoring of atrial rhythms, in particular atrial flutter and atrial fibrillation (FA). An efficient noninvasive study of the $A A$ needs the ventricular activity cancellation. The Discrete Packet Wavelet Transform (DPWT) allows the decomposition of the original ECG in a set of coefficients with different temporal and spectral features, showing that it is possible to obtain the AA with a finite set of this blocks and the inverse transform. The principal advantage of the DPWT analysis is that it does not require several leads of the same ECG register so it should be applicable to the detection of different arrhythmias in Holter registers, where the number of leads is reduced.
\end{abstract}

\section{Introduction}

One of the characteristics of the atrial fibrillation (AF) is absence of $\mathrm{P}$ waves before the QRST complex, which are replaced with base line fluctuations along the cardiac cycle. These fluctuations are known as ' $\mathrm{f}$ ' waves and they constantly change size, orientation and length. These waves are associated to an irregular cardiac frequency.

Detection and characterization of the AF in an electrocardiogram (ECG) needs a previous extraction or cancellation of the ventricular activity (VA).

Linear filtering classic techniques can not be used because of the low level of the AA and the possible spectral overlapped between the distributions associated to the ventricular and atrial signal.

The newest techniques for AA extraction -Blind Source Separation [1,2], Spatio-Temporal Cancellation $[3,4]$, Artificial Neural Networks (TDDN) [5]- show a better performance than classic techniques -like Average Beat Subtraction (AVBS) [6], Adaptative Recurrent Filtering [7-9]-, but they need several reference signals or leads in their implementation.

Until now, the Discrete Packet Wavelet Transform (DPWT) has not been applied as a non-invasive method for AA extraction.
The principal advantage of the analysis with DPWT is that it does not require several recordings of the same ECG, which is one of the disadvantages of the BSS, Spatio-Temporal and TDDN techniques. These methods have a high performance but they need two recordings at least. A brief comparison with AVBS technique, which also requires one reference signal, is done in the results section. The AVBS is based on the cancellation of each QRST complex with an average complex calculated and subtracted over the register under analysis.

\section{Theory}

The wavelet analysis has had a high development in the last years. This type of signal processing allows the joining of time and frequency analysis. It decomposes the original signal in a set of coefficients with different spectral ant time features. These coefficients are scaled and shifted versions of a function called the mother wavelet.

Implementation of the Discrete Wavelet Transform (DWT) is very easy: it can be done with a hierarchical structure decomposition [10]. It consists in several filter cascade banks that provide detail coefficients (high frequencies) and approximation coefficients (low frequencies). These coefficients have several resolutions, by using a dyadic net in the different scales. The band pass and band cut of the resulting filters sets the resolution quality of the system in every family of wavelet.

The DPWT is a generalization of the DWT and it shows a more efficient signal analysis owing to a bigger set of coefficients in all the scales and certain redundancy.

One of the most important applications of the wavelet transform is noise and interference cancellation. The generic process consists in applying a threshold to the output of the DWT and the detail or approximation coefficients below this value are eliminated. After that, the inverse transform can be applied and the signal is reconstructed without noise or interference.

There are lots of methods to reduce the noise in the ECG with wavelets $[11,12]$. The difference among them 
are the choice of the threshold and the way to apply it. Other DWT applications are the abnormal pattern identification in ECG [13-16], QRST complex detection $[17,18]$, etc.

In this paper, it is presented a variation of these classic methods of noise and interference cancellation used as an AA extraction technique.

\section{Materials}

It has been created a database of artificial signals, where the AA is known and simulated. A synthesized AF signal has been added to sinus rhythm recordings using linear combination, according to the studies and references of several authors [3], Figure 1. So, the shape and behavior of the atrial signal is known and the proposed extraction methods can be evaluated fast and efficiently.

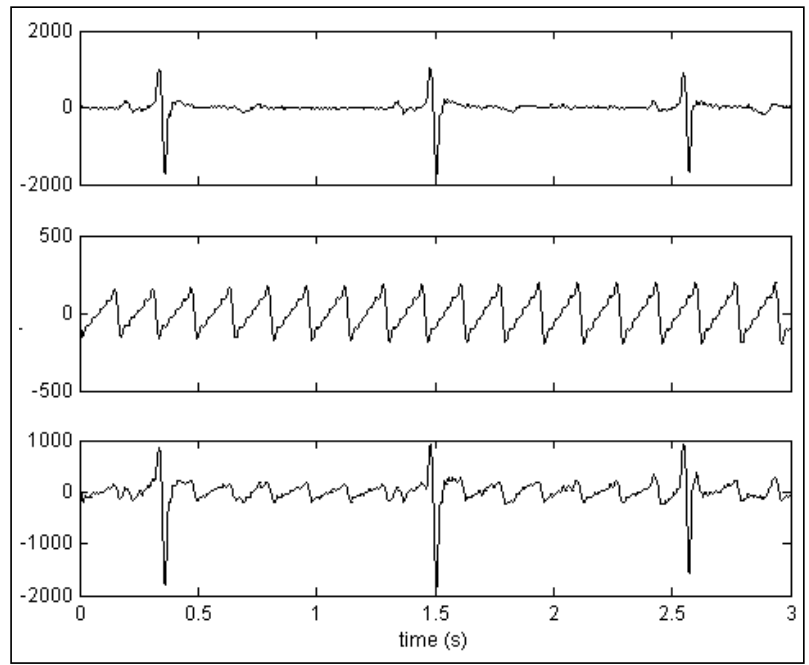

Figure. 1. Artificial AF registers. (above) Original preprocessed and normalized sinus rhythm. (middle) Simulated AF. (below) Resulting register after normalization and the lineal combination.

The used signals are created from recordings of the MIT-BIT (the Massachusetts Institute of TechnologyBeth Israel Hospital Arrhythmia Database, series 100 and 200) and an own database of ECG, with signals obtained at the Cardiac Electrophysiology Lab of the Clinic Hospital in Valencia. This database contains sinus rhythm real AF episodes and artificial AF episodes. All the registers have been pre-processed and normalized to remove possible fluctuations of the base line, interferences, noises, etc. The configuration of the database used is shown in Table 1.

Table 1. Used registers in database.

\begin{tabular}{lll}
\hline & Synthesized SR+AF & Real AF \\
\hline Number & 21 & 6 \\
\hline
\end{tabular}

\section{Method}

The basic idea in this study is to consider AF as an interference, which must be removed or extracted. So, a variation of noise cancellation techniques with DWT can be used.

Two different methodologies have been proposed and tested. Methodology 1 uses a DPWT with de-noising, applying a suitable threshold. The threshold is fixed to a value depending on the maximal values in the considered cardiac cycle. First, these peak values are localized and the mean value and standard deviation are calculated. Only the values above the $80 \%$ of the standard deviation plus the mean calculated value are considered. The final threshold is the mean of those remaining maximal multiplied by an experimental factor.

The difference between the reconstructed signal and the original ECG recording could be considered as the AA. Despite all this process, a remaining QRST complex has been observed in the obtained signals, Figure 2.

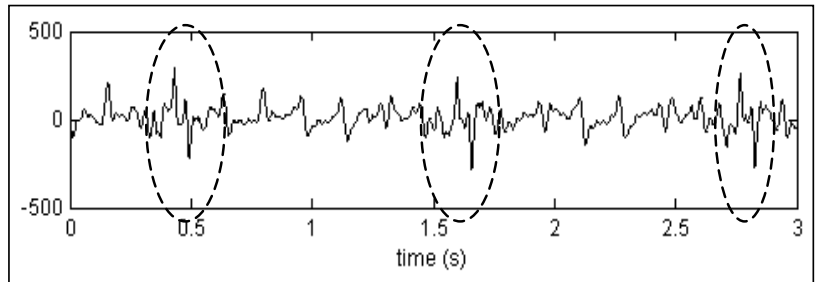

Figure 2. Remaining QRST complex in AA extracted with Methodology 1.

Methodology 2 tries to reduce this complex presence using the reconstructed signal of the previous methodology and another wavelet transform without denoising, in this case a DWT with a different wavelet function, and its associated inverse transform from the detail coefficients at level seven.

The first experimental tests established that the best specifications were the Biorthogonal 4.4. function for the first transformation with DPWT and the Daubechies 10 function for the second transformation with DWT.

\section{Results}

In all cases, the comparison between the obtained signal and the expected one is done with the cross correlation coefficients in the spectral and temporal domain. Also, others parameters have been calculated as amplitude and frequency of the main peak (fp), band peak energy and total energy distribution ratio (PPBP, where the band peak is the set of frequencies between $0.82 * \mathrm{fp}$ and $1.17 * \mathrm{fp})$, etc.

The visual comparison of the obtained signal in the two methodologies, the AVBS technique and the expected synthesized atrial activity is shown in Figure 3. 


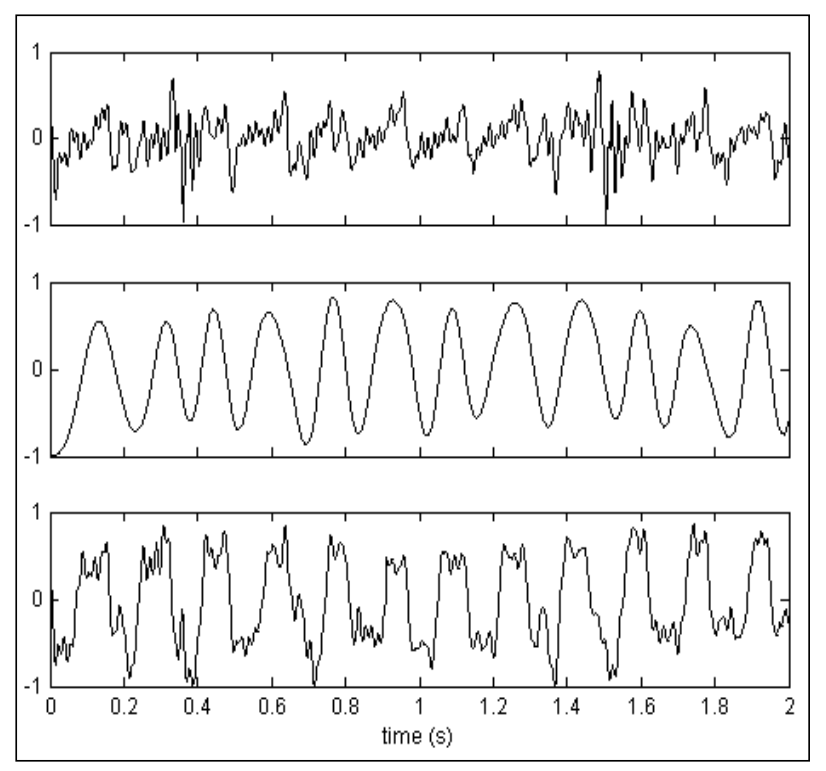

Figure 3. Comparison of extracted atrial signal for episode of Fig. 1 via (above) Methodology 1, (middle) Methodology 2 and (below) AVBS method.

In the real $\mathrm{AF}$ signals there are not reference atrial activity to calculate the correlation values. Other parameters have been considered in these cases to identify the AF episode, for example spectral energy distribution. The results show an extracted AA very similar to the real atrial waveforms presented in ECG registers, as it is illustrated in Figure 4.

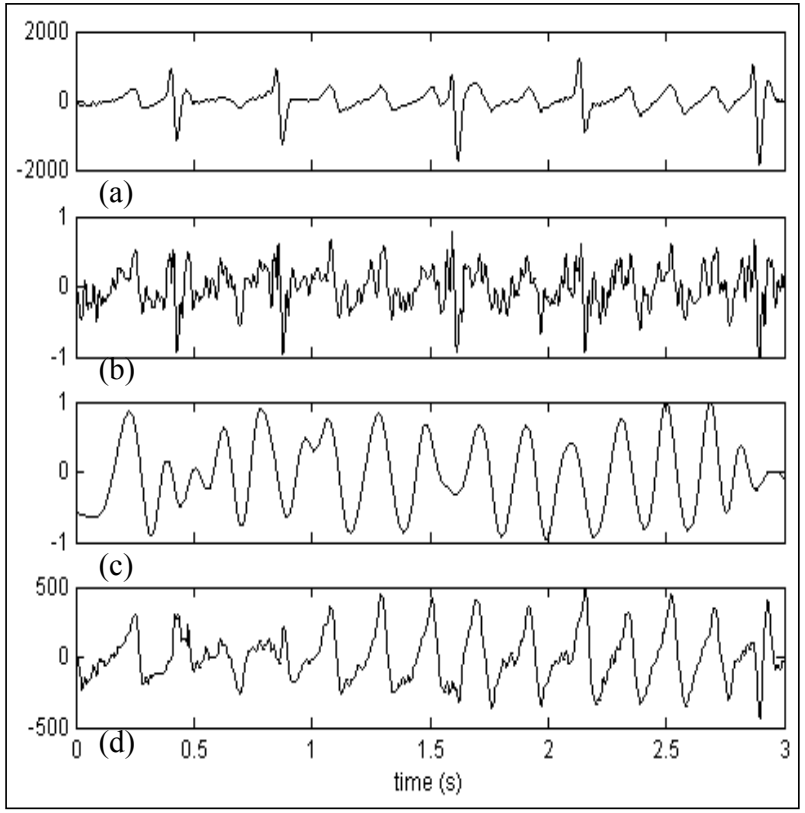

Figure 4. Comparison of normalised extracted atrial signal from a real AF episode (a) via Methodology 1 (b) Methodology 2 (c) and AVBS method (d).
These methods have been applied to the registers in Table 1 and the results are shown in Table 2, with their mean value and standard deviation $(\mathrm{p} \leq 0.05)$.

Table 2. Correlation coefficients and spectral parameters in both proposed methodologies and the AVBS method. Comparison with the synthesized AF signal parameters.

\begin{tabular}{lcc}
\hline & Method. 1 & Method. 2 \\
\hline Spectral Corr. & $0.84 \pm 0.09$ & $0.87 \pm 0.10$ \\
\hline Temporal Corr. & $0.51 \pm 0.11$ & $0.55 \pm 0.12$ \\
\hline Main Peak (Hz) & $7.56 \pm 0.91$ & $6.37 \pm 0.71$ \\
\hline PPBP Ratio & $0.57 \pm 0.13$ & $0.71 \pm 0.14$ \\
\hline
\end{tabular}

\begin{tabular}{lcc}
\hline & AVBS & Synthesized AF \\
\hline Spectral Corr. & $0.66 \pm 0.30$ & --- \\
\hline Temporal Corr. & $0.47 \pm 0.25$ & ---- \\
\hline Main Peak (Hz) & $5.91 \pm 0.67$ & 6.10 \\
\hline PPBP Ratio & $0.41 \pm 0.15$ & 0.69 \\
\hline
\end{tabular}

Methodology 2 presents satisfactory results, very similar to expected values. In the other hand, the Methodology 1 and the AVBS method show low cross correlation coefficients and low energy concentration around the principal frequency.

\section{Conclusions}

In the present work, the possibilities of the wavelet transform have been shown as an alternative atrial extraction method.

The electrocardiography registers have an implicit atrial that it is underhanded with the synthesized one. This could justify the obtained cross-correlation values.

Methodology 2 extracts a smoother signal with higher cross correlation coefficients, but it needs two consecutive transformations and it couldn't be convenient due to the increase of the computational load.

The presented results show a good similarity between the expected AA and the obtained signal. The principal peak in the $5-8 \mathrm{~Hz}$ range and the energy concentration around the band peak are characteristic of this type of arrhythmias.

Although these results do not beat the other techniques results yet, they present several advantages, for example the easy implementation, low computational load and the minimum required reference recordings of the same ECG.

So, this new technique should be applicable to arrhythmias like the atrial paroxismal fibrillation, which is usually detected with Holter systems where the number of leads is reduced.

\section{Acknowledgements}

This work was partly funded by several research 
grants from the 'European Funds for Regional Development' (1FD97-2147), the 'Department of Electric, Electronic and Automatic Engineering', the 'Department of Theory of Signal' in the University of Castilla-La Mancha and the research incentive program of the Polytechnic University of Valencia, Spain.

\section{References}

[1] Rieta J. J., Zarzoso V., Millet-Roig J., García-Civera R., Ruiz-Granell R., "Atrial Activity Extraction Based on Blind Source Separation as an Alternative to QRST Cancellation for Atrial Fibrillation Analysis". IEEE Computers in Cardiology 2000;27:69-72

[2] Zarzoso V., Nandi A.K., "Noninvasive Fetal Electrocardiogram Extraction: Blind Separation Versus Adaptive Noise Cancellation”. IEEE Trans. Biomed. Eng. 2001; 48, 12-18.

[3] Stridh M, Sörnmo L., "Spatiotemporal QRST cancellation techniques for Atrial Fibrillation analysis:methods and performance". IEEE Computers in Cardiology 1998;25:633-636.

[4] Stridh M, Sörnmo L., Meurling C.J., Olsson S.B., "Characterization of Atrial Fibrillation Using the Surface ECG: Time-Dependent Spectral Properties". IEEE. Trans. BioMed. Eng. 2001;48:19-21.

[5] Vasquez C., Hernández A., Mora F., Carrault G., Passariello G., "Atrial Activity Enhancement by Wiener Filtering Using an Artificial Network". IEEE. Trans. BioMed. Eng. 2001;48:940-944.

[6] Shkurovich S., Sahakian A.V., Swiryn S., "Detection of Atrial Activity from High-Voltage Leads of Implantable Ventricular Defibrillators Using a Cancellation Technique". IEEE Trans. Biomed. Eng. 1998;45:229-234.

[7] Sörnmo L., Pahlm O., Nygards M., "Adaptive QRS detection: A study of performance". IEEE Trans. Biomed. Eng. 1985;32:392-401.

[8] Thakor N., Yi-Zheng Z., "Applications of adaptative filtering to ECG analysis: Noise cancellation and arrhytmia detection", IEEE Trans. Biomed Eng. 1991;38:785-794.
[9] Colomer A., Terré V., "Electrocardiogram enhancement by adaptative filtering". Signal Processing VII: Theories and Applications, Proceedings of EUSIPCO-94 1994;1:58-61.

[10] Provaznik I., Kozumplik J., Bardonova J., Novakova M., Novakova Z., "Wavelet Transform. In ECG Signal Processing". Proceeding of the $15^{\text {th }}$ Biennial Eurasip Conference BIOSIGNAL 2000:21-25.

[11] Mallat S., "A Wavelet Tour of Signal Processing", Academic Press, 1998.

[12] Roberts S., Chaumeron T., Magdolen J., “Adaptive Noise Removal from Complex Signals using the Wavelet Transform". Signal Processing VII: Theories and Applications Proceedings of EUSIPCO 1994;1:66-69.

[13] Sita G., Ramakrishnam A.G., "Wavelet Domain Nonlinear Filtering for Evoked Potencial Signal Enhancement". Applied and Computational Harmonic Analysis 2001;33: 431-446.

[14] Couderc JP, Zareba W. "Contribution of wavelets to the non-invasive electrocardiology". ANE, 1998; 3:54-62.

[15] Dai W., Yang Z., Lim S. L., Mikhailova O., Chee J., "Processing and Analysis of ECG Signal Using Nonorthogonal Wavelet Transform". Proceedings $-20^{\text {th }}$ Annual International Conference. IEEE/EMBS 1998:139142.

[16] Thurner S., Feurstein M. C., Teich M. C., "Multiresolution Wavelet Analysis of Heartbeat Intervals Discriminates Helathy Patients from Those width Cardiac Pathology". Phsycal Review Letters 1998;80:1544-1547.

[17] Millet J., Ruiz García S., Ruiz-Granell R., Civera R., "Algoritmo para la detección de FV mediante la Transformada Wavelet". Proceedings XIV Congreso Anual de la Sociedad Española de Ingeniería Biomédica. 1996.

[18] Li C., Zheng C., Tai C., "Detection of ECG Characteristic Points Using Wavelet Transforms.”. IEEE. Trans. BioMed. Eng. 1995:42:21-28.

Address for correspondence.

César Sánchez Meléndez

E.U.P. Cuenca. Universidad de Castilla-La Mancha.

Campus Universitario s/n. 16071. Cuenca.

E-mail: cesar.sanchez@uclm.es 